\title{
TRASTORNOS DEL ESTADO DE ÁNIMO AL FINAL DE LA VIDA: ¿DESMORALIZACIÓN O DEPRESIÓN?
}

\author{
GemMa GARcía-SORIANO y PilAr BARRETO
}

Facultad de Psicología, Universidad de Valencia

\begin{abstract}
Resumen: Este trabajo analiza la problemática que conlleva la alteración del estado de ánimo, en concreto la tristeza en la población de enfermos físicos graves al final de la vida. Se analizan los problemas e implicaciones de un diagnóstico inadecuado, las confusiones asociadas al diagnóstico de depresión, se evidencia la importancia de distinguir entre las reacciones «normales» de malestar ante la enfermedad, y las reacciones patológicas. Así mismo se revisan y analizan distintos trabajos que proponen la incorporación de un nuevo síndrome a las clasificaciones psiquiátricas tradicionales: la desmoralización. Finalmente se discuten las implicaciones, ventajas e inconvenientes que tendría la incorporación del síndrome de desmoralización.
\end{abstract}

Palabras clave: desmoralización, depresión, final de la vida.

\section{Affective disorders at the end of life: demoralization or depression?}

\begin{abstract}
This paper analyzes problems that result in an alteration of mood, particularly in the sadness found in people with a serious physical illness at the end of life. The problems and implications of an faulty diagnosis, the confusions associated with a diagnosis of depression, and the importance of differentiation between «normal» mood reactions to illness, and pathological reactions, are analyzed. Several studies are reviewed and analyzed that propose the incorporation of a new syndrome to the traditional psychiatric classifications: demoralization. Finally the implications, advantages, and inconveniences of including a demoralization syndrome are discussed.
\end{abstract}

Keywords: demoralization, depression, end of life.

\section{INTRODUCCIÓN}

La presencia de depresión, así como de otras alteraciones de tipo afectivo, dificulta la consecución del objetivo último de los cuidados paliativos (Doyle, Hanks, y McDonald, 1993), que supone la consecución de la máxima calidad de vida posible para los pacientes y sus allegados.

Dada la confusión existente en la literatura actual sobre el diagnóstico de las alteraciones de tipo afectivo en pacientes con enfermedades físicas en la fase final de la vida, y con el objetivo de colaborar en el esclarecimiento del estudio del estado de ánimo triste y su patología en

Recibido 8 septiembre 2006; aceptado 6 febrero 2007

Correspondencia: Gemma García Soriano, Departamento de Personalidad, Evaluación y Tratamientos Psicológicos, Facultad de Psicología, Universidad de Valencia, Avda. Blasco Ibáñez, 21, 46010 Valencia. Correo-e: Gemma.Garcia@uv.es esta población, nos hemos propuesto realizar una revisión de los últimos trabajos enmarcados en esta área de conocimiento.

Comencemos por establecer algunos puntos de partida. La literatura científica señala que:

(a) Más del 60\% de los pacientes con cáncer informan padecer malestar afectivo. En un trabajo (Derogatis, Morrow, Fetting, Penman, Piasetsky, y Schmale, 1983) realizado con una muestra de pacientes en diferentes estadios del cáncer se encontró que el $47 \%$ de los sujetos cumplían criterios para algún trastorno psiquiátrico: un $68 \%$ de los casos presentaban estado de ánimo ansioso o depresivo; un $13 \%$ cumplía los criterios de trastorno depresivo mayor, y un $8 \%$ padecía trastornos mentales orgánicos como delirium. La investigación indica que pacientes con otras enfermedades en la fase final también presentan una mayor incidencia 
de trastornos psiquiátricos que la población sana (Cassem, 1995).

(b) La tristeza asociada al síndrome depresivo disminuye la calidad de vida de los pacientes y las interacciones con la familia y amigos. Así mismo, reduce la efectividad del tratamiento de los síntomas físicos y del control del dolor, suponiendo una fuente adicional de estrés. En poblaciones clínicas, los pacientes con depresión tienen alteraciones en la funcionalidad física similares a las de los pacientes con trastornos físicos graves como la artritis. Y a nivel social, sus limitaciones son mayores (Hotopf, Chidgey, Addington-Hall, y Lan Ly, 2002). También es importante diferenciar entre la reacción normal de tristeza adaptativa al final de la vida debida a las sucesivas pérdidas y decepciones que se tienen que afrontar y la depresión clínica. Es habitual que la tristeza se encuentre acompañada por sentimientos de baja autoestima, desánimo, aflicción, abatimiento y en ocasiones ideas de culpa e indignidad. Puede ir acompañada de la inhibición de la expresión facial y de los movimientos, así como de un progresivo aislamiento del sujeto, que cada vez será menos comunicativo y tolerante, pudiendo llegar al rechazo, generalmente pasivo, de los demás.

(c) El diagnóstico y tratamiento adecuado de la depresión tiene implicaciones sobre el deseo de acelerar la propia muerte, y sobre el suicidio asistido en aquellos países donde está permitido. En un trabajo empírico, Breitbart (2000) encuentra relaciones significativas entre la depresión en pacientes de cáncer al final de la vida diagnosticados con la SCID (Structured Clinical Interview for DSM-IV; Spitzer, Gibbon, y Williams, 1996) y el deseo de acelerar la muerte. Los pacientes con depresión mayor tenían cuatro veces más posibilidades de desear la muerte acelerada que los que no tenían este trastorno ( $47 \%$ vs. $12 \%)$.

Así pues, el avance en el conocimiento sobre el diagnóstico adecuado del estado de ánimo patológico en la fase final de la vida reviste gran importancia. Solo mejoras de este tipo permitirán avanzar en la planificación de programas de intervención adecuados que incidan en la disminución del sufrimiento del paciente y sus familiares.

\section{LA DEPRESIÓN EN LOS ENFERMOS EN LA FASE FINAL DE LA VIDA}

\section{Dificultades diagnósticas}

Se han empleado diversos criterios diagnósticos para identificar a los pacientes con depresión mayor, siendo los más comunes los del DSM-IV y los Criterios Diagnósticos de Investigación (Research Diagnostic Criteria, RDC) (Spitzer, Endicott, y Robins, 1978). La prevalencia de pacientes diagnosticados de depresión mayor varía en función del sistema diagnóstico empleado, inconsistencia especialmente relevante cuando la depresión se asocia a enfermedad médica grave, encontrándose variaciones tan llamativas como entre un 2 y un $45 \%$ (Noyes y Kathol, 1986), y entre un 1 y un 53\% (Breitbart, 1987).

La dificultad de diagnosticar el trastorno depresivo en pacientes con cáncer u otras enfermedades graves ha sido documentada en diferentes trabajos (Brugha, 1993; Chochinov, Wilson, Enns, y Lander, 1994, 1997; LoydWilliams y Payne, 2002; Passik, et al., 1998). Todos ellos coinciden en considerar como implicados factores relativos al: (1) personal sanitario (p.ej., empleo de indicadores poco fiables de la depresión, sobrevaloración de la sintomatología somática, desconocimiento de los sentimientos de los pacientes, tendencia a sobrevalorar el malestar del paciente, actitudes negativas hacia el diagnóstico de depresión, etc.); (2) a las características de los pacientes (p.ej., expresión de bloqueo, dificultad para informar de sus síntomas depresivos, resistencia a buscar tratamiento por sus síntomas psicológicos); (3) a las características de la enfermedad (p.ej., dificultad para distinguir entre los síntomas somáticos que son consecuencia de la enfermedad y los que lo son de la depresión); (4) características de la depresión (p.ej., dificultad para distinguir entre depresión clínica y disforia subclínica o respuesta «normal» a una situación estresante); (5) condiciones sanitarias (p.ej., elevado número de pacientes por médico, carencia de estructuras adecuadas para discutir los problemas personales y emocionales). 
La depresión clínica en enfermos en la fase final de la vida

A pesar del apoyo empírico y la insistencia por parte de distintos autores (Endicott, 1984; Kathol, Mutgi, Williams, Clamon, y Noyes, 1990) de que la depresión en pacientes con enfermedad avanzada requiere criterios especiales, el DSM-IV-TR (APA, 2000) sigue sin considerar un diagnóstico específico o diferencial para este tipo de pacientes.

Block (2000) sugiere algunos de los síntomas a tomar en consideración para el diagnóstico adecuado de la depresión en estos pacientes:

(1) Síntomas psicológicos: disforia, estado de ánimo depresivo, tristeza, sentimientos de impotencia, desesperanza, aislamiento social, culpabilidad, ideación suicida, llanto intenso, anhedonia;

(2) indicadores de la historia familiar de abuso de sustancias, depresión, trastorno bipolar o cáncer de páncreas;

(3) otros indicadores: dolor u otros síntomas intratables, discapacidad desproporcionada, preocupación somática excesiva, tratamiento con corticoesteroides, escasa cooperación o rechazo del tratamiento,

(4) reacción que generan en los demás: irradiación del afecto de aburrimiento, desesperanza, aversión y poco interés en los demás

\section{Diagnóstico diferencial}

Es importante distinguir entre la depresión como patología y la pena como estado más transitorio (véase Tabla 1) y entre la desesperanza y la depresión. Estos dos últimos diagnósticos contribuyen al deseo de una muerte acelerada, pero de forma independiente. Así, los sujetos con depresión pueden manifestar desesperanza como síntoma, pero la desesperanza por sí misma no implica padecer depresión (Breitbart et al., 2000).

\section{Evaluación de la depresión}

La depresión en esta población se ha evaluado con diferentes herramientas, siendo las más frecuentes:

Versión abreviada de la Escala de Trastornos Afectivos y Esquizofrenia (SADS; Endicott y Spizer, 1978). Incluye ítems referidos al diagnóstico de los episodios de depresión mayor y distimia, de acuerdo con los RDC y los criterios de sustitución de Endicott.

Escala de depresión postnatal de Edimburgo (EPDS, Cox, Holden, y Sagovsky, 1987). Escala de 10 ítems, con un punto de corte de 13 puntos para identificar depresión. Incluye ítems relacionados con la tristeza subjetiva, la culpa, la desesperanza, y los sentimientos autopunitivos, siendo éstos independientes de la discapacidad física. Validada en una muestra de 100 pacientes con cáncer avanzado (LloydWilliams, Friedman, y Rudd, 2000).

Escala de Ansiedad y Depresión Hospitalaria (HADS; Silverstone, 1994). Desarrollada para diagnosticar ansiedad y depresión en población médica general, aunque a menudo se ha emplea-

Tabla 1. Características diferenciales entre la depresión y la pena

\begin{tabular}{lll}
\hline & \multicolumn{1}{c}{ Depresión } & \multicolumn{1}{c}{ Pena } \\
\hline Origen & aspectos generalizados de la vida & pérdida particular \\
Sintomatología & somática y cognitiva & somática \\
Evolución & constante y progresiva & a «oleadas» \\
Ideación suicida & intensa y persistente & deseos pasivos de una muerte rápida \\
Visión de futuro & incapacidad ver futuro positivo & mantienen esperanzas \\
Tratamiento & necesario siempre & solo en ocasiones \\
Prevalencia & $1-53 \%$ & mayoría de personas en la fase final de \\
\hline
\end{tabular}


do en cuidados paliativos. Inconsistencia de resultados en los trabajos que han estudiado su idoneidad como prueba de screening en pacientes con cáncer avanzado (Lloyd-Williams, Friedman, y Rudd, 2001; Le Fevre, Devereux, Smith et al., 1999; Holtom y Barraclough, 2000).

Entrevista de un item, y entrevista de dos items (Chochinov y Wilson, 1998; Chochinov, Wilson, Enns, y Lander, 1997). En la primera se pregunta al sujeto si está deprimido, y en la segunda se añade una pregunta sobre la pérdida de intereses. Estas dos preguntas cubren el núcleo de los criterios de la depresión del DSMIV-TR (APA, 2000), y sirven como una primera aproximación a la posible existencia de depresión en los enfermos graves. Los autores hallaron una elevada fiabilidad y discriminación, detectando menos falsos positivos que el BDI (Inventario de Depresión de Beck), y una escala análoga visual (Chochinov y Wilson, 1998)

En un trabajo reciente de revisión, Hotopf et al. (2002) tras identificar cuatro aproximaciones empleadas para identificar la depresión en pacientes al final de la vida (criterio clínico; cuestionarios de un ítem; cuestionarios como el HADS, o el BDI, y entrevistas diagnósticas), concluyeron que los niveles de prevalencia de la depresión empleando criterios de sustitución no diferían de las descripciones convencionales de la misma.

\section{EL SÍNDROME DE DESMORALIZACIÓN: UNA ALTERNATIVA DIAGNÓSTICA}

En las últimas décadas se ha reconocido la existencia de «un estado» que puede ser confundido con el diagnóstico de depresión (sobre el que volveremos más adelante), pero que hace referencia a algo más abstracto, más espiritual, un dolor emocional profundo, una pérdida de significado, o de esperanza. Kissane, Clarke, y Street (2001) en un artículo de revisión, señalan que éstas son características peculiares del enfermo en la fase final de la vida, y proponen un término que las engloba mejor que el problemático diagnóstico de la depresión: el síndrome de desmoralización.

\section{Conceptualización}

La desmoralización ha sido reconocida de forma independiente en diferentes contextos clínicos. Frank (1973) la conceptualizó como «un fallo persistente en el manejo del estrés, asociado a sentimientos de impotencia, desesperación, incompetencia personal y soledad», como un estado en el que «la autoestima suele estar dañada, la persona ha perdido el sentido de la vida, y cuando no cumple las expectativas de los otros, surge un sentimiento de alienación», y la propuso como denominador común a todas las condiciones que la psicoterapia trata de aliviar o resolver. Por su parte, Engel (1985), en una muestra de pacientes no psiquiátricos reconoce un estado semejante a la desmoralización, que llama «complejo de abandono o renuncia del paciente» ('giving up-given up'). En esta misma dirección, Gruenberg (1967) acuñó el nombre de «síndrome de fracaso social» ('social breakdown syndrome') para referirse a lo que parece una desmoralización crónica en pacientes con desorden mental prolongado, y Cassell (1982) describió el «sufrimiento» ('suffering') como un dolor experimentado por las personas, y no solo los cuerpos, cuyo origen está en los retos que amenazan la integridad de la persona como entidad social y psicológica.

El término desmoralización se ha empleado al menos desde tres perspectivas diferentes (De Figueiredo, 1993): como estrés «no específico», como la incapacidad autopercibida para hacer frente a una situación estresante determinada, y finalmente como una síntesis de las dos posiciones anteriores, a saber, como una combinación de malestar y de incompetencia subjetiva.

Desde la primera perspectiva se ha propuesto que el término desmoralización engloba el malestar psicológico no específico que los estudios epidemiológicos han reconocido en los enfermos al final de la vida (Dohrenwend, Shrout, Ergi, y Mendelsohn, 1980). Se define en este caso como una constelación de sentimientos entre los que se encuentran la baja autoestima, la desesperanza, el desamparo, la confusión de pensamiento, el miedo a perder el control y volverse loco, la ansiedad, la tristeza, 
algunos síntomas psicofísicos, y percepciones de pobre salud física. Los autores señalan que comparte características con la depresión, pero que sus respuestas son menos severas, considerando este síndrome como una dimensión más de la Salud Mental, e insistiendo en la importancia de diagnosticar la desmoralización, puesto que incluso en ausencia de un trastorno clínico, es un importante factor de malestar que puede incrementar el sufrimiento que acompaña a diferentes problemas.

La segunda visión (Klein, Gittelman, Quitkin, y Rifkin, 1980) define la desmoralización como la incapacidad o incompetencia percibida de afrontar de forma efectiva una situación estresante. Característica que la distinguiría de la depresión: el sujeto con desmoralización no puede disfrutar del placer anticipatorio, pero sí del consumatorio, mientras que la persona con depresión será incapaz de sentir placer en cualquiera de los dos casos.

Desde la última perspectiva se define la desmoralización como una combinación de males$\operatorname{tar}$ (p.ej., ansiedad, tristeza, enfado, o resentimiento), e incompetencia subjetiva (percepción del sujeto de que es incapaz de actuar y expresar los sentimientos que considera apropiados en una situación estresante). Los autores (De Figueiredo y Frank, 1982) señalan que el malestar y la incompetencia percibida tienden a coexistir cuando las asunciones importantes para la autoestima se confirman, o cuando los vínculos sociales del sujeto son inadecuados.

Recientemente, Clarke y Kissane (2002) se han decantado por esta última aproximación, aunque disienten de Frank en que la desmoralización sea simplemente un malestar no específico, para ellos, se trata de un síndrome claramente definido de estrés existencial, que ocurre en pacientes con enfermedad física o mental, concretamente sobre aquellos que sienten amenazada su vida o integridad física.

Por último parece importante reflejar el planteamiento de Rickelman (2002), quien ha propuesto una definición teórica de la desmoralización como parte de un continuo de respuestas depresógenas, que precedería e incrementaría el riesgo del trastorno depresivo mayor. Este autor une dos tipos de factores: los cognitivos, que en diferentes grados juegan un rol tanto en la depresión como en la desmoralización (estilo atribucional, impotencia, percepción de ausencia de control, pesimismo, evitación de responsabilidad, incertidumbre en la toma de decisiones, y rigidez cognitiva) y los personales-ambientales, que reflejan la interacción entre el sujeto y el ambiente.

Según este modelo, las personas estarían desmoralizadas, en lugar de deprimidas, cuando percibieran que al menos temporalmente, no pueden responder a las demandas de estresores vitales específicos o bien cuando atribuyen sus fallos a déficits, tanto externos como internos, y manifiestan problemas de afrontamiento en sus respuestas cognitivas, afectivas y sociales. En este sentido, cuanto más globales y estables sean las atribuciones negativas del sujeto para sus sucesos estresantes vitales, será más probable que se agraven sus síntomas depresivos.

Finalmente se ha de señalar que la desmoralización se ha estudiado asociada a los cuidados paliativos, a la enfermedad en fase final, a la enfermedad médica crónica, a la minusvalía física, o a la desfiguración del cuerpo, y en grupos como los ancianos, cuidadores de niños con minusvalías, enfermos internados en residencias, familiares de enfermos terminales (esposas e hijos) (Gurland y Toner, 1982), o personas que viven solas vs. en compañía (Page y Colle, 1992).

En definitiva, la desmoralización es un término que durante las últimas cuatro décadas, y desde distintos contextos, se ha venido aplicando a distintas poblaciones para referirse a un conjunto de síntomas subjetivos de malestar e incompetencia para hacer frente a distintas situaciones de la vida cotidiana. No obstante no ha sido recogido por los sistemas de clasificación psiquiátricos.

\section{Características del sindrome de desmoralización en el enfermo en la fase final de la vida}

En el ámbito que nos ocupa, la desmoralización se ha considerado como un estado psíquico cuyos pilares básicos son la desesperanza (pérdida de esperanza generalizada -rasgo-, y espe- 
cífica - estado-), la impotencia, la pérdida de significado, el malestar existencial, el pesimismo, algún grado de soledad, y deterioro en el afrontamiento. Este último, centrado en el problema vs. en la emoción, regula el malestar, de modo que cuando los mecanismos de afrontamiento son insuficientes, y el sujeto no sabe qué hacer, se originan un gran malestar y se experimenta impotencia (Clarke y Kissane, 2002)

Otros autores (Fava, Freyberger, Bech, Christodoulou, Sensky, y Theorell, 1995), ahondando en los planteamientos de Schmale (1972), describen la desmoralización como una toma de conciencia prolongada de no cumplir con las expectativas o manejo de los problemas, que provoca sentimientos de impotencia, desesperanza o abandono. Estos autores proponen que para su diagnóstico, el sentimiento debe persistir por lo menos un mes asociado a algún trastorno médico, y en ausencia de otro trastorno mental.

En este mismo sentido, Kissane y Kelly (2000) definen la desmoralización como un síndrome clínico específico de las enfermedades médicas, y proponen los siguientes seis criterios diagnósticos con persistencia mínima de dos semanas: (1) Síntomas afectivos de angustia existencial, incluyendo desesperanza o pérdida de significado y sentido de la vida; (2) actitudes cognitivas de pesimismo, impotencia, sensación de estar atrapado, de fracaso personal o pérdida de un futuro con significado; (3) ausencia de motivación; (4) aislamiento social/ausencia de apoyo social; (5) fluctuación del estado emocional durante más de dos semanas, y (6) ausencia de depresión mayor y de cualquier otro trastorno psiquiátrico como condición primaria.

Otros autores especifican que a pese a los síntomas afectivos negativos, los sujetos con síndrome de desmoralización mantienen la capacidad de interactuar con el ambiente y de disfrutar el presente, aunque sea sin entusiasmo.

Respecto a los síntomas específicos, la impotencia y el sentimiento de incompetencia que conlleva la desmoralización, a menudo inducen a desear la muerte o el suicidio debido a la incapacidad de encontrar sentido a la vida, y no con un sentimiento de realización personal como puede ocurrir en la vejez (Kissane, 1998). A este respecto, Kelly y Robertson (2002) encontraron que la desmoralización se da especialmente entre los pacientes de cáncer avanzado con un mayor deseo de acelerar su muerte, por lo que defienden la necesidad de investigar el rol del síndrome de desmoralización en las peticiones de suicidio, y de considerarlo como un foco potencial de intervenciones clínicas.

Aunque en principio el grupo de Kissane (2001) excluye el diagnóstico de desmoralización si existe trastorno depresivo mayor, en un trabajo posterior (Clarke y Kissane, 2002) señalan que esta es una solución pragmática que no representa adecuadamente la observación clínica de que la desmoralización puede coexistir tanto con la depresión, como con el cáncer, la esquizofrenia, $\mathrm{u}$ otros trastornos.

Finalmente señalar que este síndrome se da con mayor prevalencia en las mujeres, tal Kissane (2001) apunta que este hecho puede deberse a su mayor necesidad de un rol social significativo, lo que no deja de ser una especulación teórica sin apoyatura empírica.

Diagnóstico diferencial en el sindrome de desmoralización

La desmoralización, igual que ocurre con la depresión, suele pasar desapercibida en los enfermos en fase avanzada debido al deterioro que conlleva la enfermedad, también se le resta importancia señalando que dada la situación de los pacientes, es comprensible y racional que presenten estos sentimientos de desesperanza y estrés emocional.

El grupo de Kissane (2001), además de proponer la existencia del síndrome, plantean la necesidad de diferenciarla de otros fenómenos como: la abulia (asociada a una pérdida neurológica de motivación), y la apatía: en el síndrome de desmoralización, la baja motivación es importante pero no es rasgo principal como en estos dos síntomas; también del aburrimiento: definido como un sentimiento derivado de la monotonía sensorial y reducción de la vida interna e imaginación; y de la vergüenza. Respecto a esta última, hemos de señalar que son necesarios estudios que la relacionen con la desmoralización, los que se han efectuado hasta el momento la relacionan con el suicidio y el rechazo del cuerpo, pero no con la depresión (Gilbert, Pehl, y Allan, 1994). 
Otros autores (De Figueiredo y Frank, 1982) señalan la importancia de distinguir entre uno de los factores que componen la desmoralización, la incompetencia subjetiva, y otros conceptos como el «mastery» de Caplan, el «locus de control» de Rotter, la «desesperanza aprendida» de Seligman, la «autoeficacia» de Bandura, o el «neuroticismo» de Eysenck.

Pero si de algún fenómeno es interesante diferenciar la desmoralización, es de la depresión puesto que en este caso se trata de afinar en el diagnóstico, y tal como hemos comentado anteriormente, se ha llegado a plantear que se trata de un mismo trastorno con menor intensidad.

Entre la sintomatología diferencial que se ha venido señalando, destaca el sentimiento de impotencia y pérdida de sentido y significado de la vida de la persona con desmoralización, sentimientos que a menudo les conducirán a que a pesar de su capacidad para disfrutar del presente sean incapaces de anticipar un futuro placentero. En cambio, debido a la anhedonia característica de los sujetos deprimidos, éstos serán incapaces tanto de disfrutar del presente como de anticipar placer futuro. Dada la pérdida de motivación, el sujeto deprimido, a pesar de que conoce cómo actuar, no lo hará; en cambio, el desmoralizado no actuará porque no sabe qué hacer. Es necesario señalar que esta distinción entre la capacidad hedónica y la desmoralización ha encontrado apoyo empírico en diferentes trabajos (p.ej., Clarke y Kissane, 2002).

Dos trabajos realizados con muestras de enfermos al final de la vida, especialmente oncológicos (Clarke, Mackinnon, Smith, McKenzie, y Hermna, 2000; Passik, Dugan, McDonald, Rosenfeld, Theobald, y Edgerton, 1998) encuentran apoyo a la idea de que la depresión anhedónica, la desmoralización, y la pena son dimensiones completamente independientes.

\section{Evaluación de la desmoralización}

Para la evaluación de este fenómeno se ha propuesto un inventario extraído del Psychiatric Epidemiology Research Interview (PERI): el PERI Desmoralization Composite. Consta de 26 ítems (Zatura, Guarnaccia, y Reich, 1988) que representan sentimientos de desmoralización, y son consistentes con la definición de desmoralización de Frank (1973), correspondiéndose con las 8 subescalas del PERI que miden «estrés psicológico no específico» (Dohrenwend et al., 1980): baja autoestima, desesperanza, pensamiento confuso, pavor (miedo a perder el control y volverse loco), ansiedad, tristeza, síntomas psicofísicos, y percepciones de pobre salud física.

\section{La desmoralización en las clasificaciones psiquiátricas}

La desmoralización es un trastorno que no aparece en el DSM-IV-TR (APA, 2000), pero que podría, en su caso, incluirse dentro del trastorno adaptativo (Kisanne et al., 2001). Distintos autores han propuesto diferentes ubicaciones para la desmoralización:

Slavney (1999) considera la desmoralización como una respuesta normal a la adversidad, y señala que se podría incluir en el código $\mathrm{V}$ del DSM, opción que minimizaría la importancia del síndrome.

Tanto De Figueiredo (1998) como Clarke y Kissane (2002) consideran que la desmoralización siempre es «anormal», por lo que descartan la propuesta de Slavney. El primer grupo propone ubicarla en el eje IV, identificándola con estresores psicosociales y ambientales; opción que la sitúa como una alteración reactiva, mientras que Clarke y Kissane proponen situarla en el eje I. Esta opción es más acorde con la definición más ampliamente referida a lo largo de este trabajo, según la cual el síndrome sí está relacionado con eventos internos del individuo. Los síntomas del estado de ánimo, esperanza y competencia subjetiva serían básicos e internos.

Recientemente se ha realizado una nueva propuesta (Clarke, Smith, Dowe, y McKenzie, 2003) de clasificación de la psicopatología experimentada por los enfermos médicos (empleando una muestra de enfermos cardiovasculares, gastrointestinales, respiratorios, reumatológicos y neurológicos). Esta clasificación 
alternativa al DSM-IV-TR (APA, 2000) incluye cinco síndromes, entre los que destacan la desmoralización y la pena con desmoralización, asociadas a la depresión y formas crónicas de trastornos de somatización del DSM-IV-TR (APA, 2000). La desmoralización se describe aquí como asociada a un elevado estrés y una baja atribución de pérdida, y esta última característica la diferenciaría del síndrome de desmoralización con pena. Señalan además que no estaría relacionada con la anhedonia, y que la sufrirían en mayor medida las personas más jóvenes, con historia previa psiquiátrica, y con estresores adicionales a los propios de la enfermedad.

\section{DISCUSIÓN SOBRE LA PERTINENCIA DE AMBOS SÍNDROMES}

El hecho de no distinguir y diagnosticar el síndrome de desmoralización puede llevar a que el grupo de pacientes que sufren un sentimiento de pérdida de sentido o de esperanza, no sea detectado ni tratado, pudiendo desembocar en una depresión. Además, puede que debido a su desesperanza y pérdida de sentido, elijan una mayor aceleración de la muerte, y al no ser diagnosticados, los clínicos pueden confundir esta decisión tomada desde su visión distorsionada de una vida en la que se sienten atrapados, como una decisión racional.

Muchos clínicos son reacios a realizar el diagnóstico de desmoralización en pacientes en fase final. Algunos consideran que la etiqueta de un diagnóstico psiquiátrico supone un gran estigma, algo que no merecen los pacientes en esta condición, y sin embargo, otros coinciden en que solo es necesario dar apoyo a las «necesidades patológicas», siendo los síntomas de desmoralización normales en cualquier persona que se encuentre en la situación de los enfermos en la fase final de la vida. En todo caso, la no identificación del trastorno, conllevará que ni se trate ni se proporcione apoyo al paciente.

Reconocer la desmoralización como un síndrome diferente de la depresión, y no sólo trabajar con los conceptos de esperanza, desesperación y significado de la vida de forma individual, permitirá: (1) aplicar un tratamien- to adecuado al alcanzar un diagnóstico más preciso, (2) identificar a quienes no sufren depresión pero si desmoralización, (3) tratar a quienes presentan ambas anomalías, (4) evitar que un síndrome de desmoralización no tratado derive en una depresión, (5) que cualquier tipo de malestar sea detectado, y por último, (6) que los profesionales de la salud sean entrenados en su detección, tratamiento y prevención

Pese a las evidencias empíricas (Clarke et al., 2000; Passik et al., 1998) que apoyan la existencia de un trastorno de desmoralización diferente de otras alteraciones del estado de ánimo, algunos investigadores niegan la utilidad del síndrome de desmoralización, considerándola una respuesta natural a una situación difícil. Slavney (1999) ha insistido en la necesidad de diferenciar la desmoralización del trastorno de adaptación del DSM-IV-TR (APA, 2000), pues siguiendo el criterio B para el diagnóstico del trastorno de adaptación señala que los «síntomas o comportamientos se expresan clínicamente por un malestar mayor de lo esperable en respuesta al estresor y/o por un deterioro significativo de la actividad social o laboral». Este autor señala la dificultad de determinar qué es una reacción mayor de lo esperable, y afirma que no es anormal sentir malestar cuando los sujetos están gravemente enfermos y separados de sus familias, cuando les dicen que van a estar bajo un prolongado y doloroso tratamiento, o cuando se enteran de que tienen una enfermedad terminal.

Como ya hemos señalado, la propuesta de Slavney (1999) sería codificar la desmoralización en el eje V del DSM-IV, tal y como se hace con la pérdida, que se define como una reacción no patológica al estrés que «debe ser foco de atención clínica». Si esto se hiciera con la desmoralización, se facilitaría el reconocimiento de una reacción que con frecuencia requiere intervención médica y/o psicológica, aunque esta codificación producirá otro tipo de problemas (p.ej., compañías de seguros). El citado autor concluye que la identificación errónea de la desmoralización como un trastorno psiquiátrico cambiará el peso del médico al psiquiatra, y lo que el desmoralizado necesita de su médico es un mayor compromiso.

Así pues, son necesarios un mayor número de estudios que delimiten cuándo los sentimien- 
tos descritos por el síndrome de desmoralización son normales, y cuándo son patológicos.

\section{CONCLUSIONES}

Ante la problemática de realizar un adecuado diagnóstico de depresión en pacientes con enfermedad física grave, las últimas tendencias plantean si el esfuerzo realizado por encontrar los criterios diagnósticos adecuados no se ha encauzado por el mejor camino. Tal vez hubiera sido más correcto crear un nuevo término, en este caso, un síndrome, que englobara las características específicas de los pacientes en la fase final de la vida.

Al buscar criterios de depresión ajustados al paciente terminal, se ha partido de las clasificaciones del APA, donde los síntomas somáticos son tan importantes como los cognitivos. Lo que aquí se plantea, es si no sería más adecuado crear unos nuevos criterios que englobaran características de las que hay evidencia empírica que se dan en este tipo de pacientes. Donde la desesperanza, o los sentimientos de dependencia, aspectos estos más vivenciales, más cognitivos, más emocionales, se perfilan como más determinantes que la pérdida de peso o el insomnio, tanto en la descripción de su estado de ánimo como en el diagnóstico.

Así mismo, se ha señalado la importancia de no considerar como «normal» cualquier manifestación de tristeza en cualquier grado, deberemos tener en cuenta el contexto en que se dan los síntomas, su adecuación, así como la gravedad de los mismos. Si fuera de otro modo, dejaríamos sin tratar a muchas personas que sufren.

Por último, señalar la importancia y la necesidad de seguir trabajando en esta línea. Resultan necesarias las aportaciones de estudios empíricos que permitan unificar criterios y mejorar así nuestra atención al paciente en la fase final de su vida.

Aunque comprendemos las precauciones de algunos autores ante la protesta de considerar la desmoralización como una categoría diagnóstica, consideramos imprescindible profundizar en su estudio, ya que trabajos previos a los que aquí se ha hecho referencia, muestran su existencia, y especialmente porque su no detección redundaría en el no tratamiento del enfermo y por tanto en el alejamiento del objetivo fundamental de una mejor calidad de vida para la persona hasta el momento final.

\section{REFERENCIAS}

American Psychiatric Association (2000). Diagnostic and statistical manual of mental disorders. (Text revised). Washington, DC: APA.

Block, S.D. (2000). Assessing and managing depression in the terminally ill patient. ACP-ASIM End-of-Life Care Annals of Internal Medicine, 132, 209-18.

Breitbart, W., Rosenfeld, B., Pessin, H., Kaim, M., Funesti-Esch, J., Galietta, M., Nelson, C.J., y Brescia, R. (2000). Depression, hopelessness, and desire for hastened death in terminally ill patients with cancer. JAMA: Journal of the American Medical Association, 284, 2907-2911.

Brugha, T. (1993). Depression in the terminally ill. British Journal of Hospital Medicine, 50, 175-181.

Cassell, E.J. (1982). The nature of suffering and the goals of medicine. New England Journal of Medicine, 306, 639-645.

Cassem, E.H. (1995). Depressive disorders in the medically ill An overview. Psychosomatics, 36, S2-S10.

Chochinov, H.M., Wilson, K.G., Enns, M., y Lander, S. (1997). Are you depressed? Screening for depression in the Terminally Ill. American Journal of Psychiatry, 154, 674-676.

Chochinov, H.M., Wilson, K.G., Enns, M., y Lander, S. (1994). Prevalence of Depression in the Terminally Ill: effects od diagnostic criteria and symptom threshold judgments. American Journal of Psychiatry, 151, 537-540.

Chochinov, M.D., y Wilson, K.G. (1998). Are you depressed? Screening for depression in the Terminally Ill. Reply. American Journal of Psychiatry, 155, 994995.

Clarke, D.M., y Kissane, D.W. (2002). Demoralization: its phenomenology and importance. Australian and New Zealand Journal of Psychiatry, 36, 733-742.

Clarke, D.M., Mackinnon, A.J., Smith, G.C., McKenzie, D.P., y Hermna, H.E. (2000). Dimensions of psychopatology in the medically ill: a latent trait analysis. Psychosomatics, 41, 418-425.

Clarke, D.M., Smith, G.C., Dowe, D.L., y McKenzie, D.P. (2003). An empirically derived taxonomy of common distress syndromes in the medically ill. Journal of Psychosomatic Research, 54, 323-330.

Cox, J., Holden, J., y Sagovsky, R. (1987). Detection of postnatal depression: development of 10 item Edinburgh Postnatal Depression Scale. British Journal of Psychiatry, 150, 782-786. 
De Figueiredo, J.M., y Frank, J.D. (1982). Subjective Incompetence, the Clinical Hallmark of Demoralization. Comprehensive Psychiatry, 23, 353-363.

De Figueiro, J.M. (1993). Depression and Demoralization: Phenomenologic Differences and Research Perspectives. Comprehensive Psychiatry, 34, 308-311.

Derogatis, L.R., Morrow, G.R., Fetting, J., Penman, D., Piasetsky, S., y Schmale, A.M. (1983). The prevalence of psychiatric disorders among cancer patients. JAMA: Journal of the American Medical Association, 249, 751-7.

Dohrenwend, B.P., Shrout, P.E., Ergi, G., y Mendelsonh, F.S. (1980). Nonspecific Psychological Distress and Other Dimensions of Psychopathology. Archives of General Psychiatry, 37, 1229-1236.

Doyle, D., Hanks, G.W.C., y MacDonald, N. (Ed.). (1993). Oxford textbook of palliative medicine. Oxford: Oxford University Press.

Endicott, J., y Spizer, R.L. (1978). A diagnostic interview: the Schedule for Affective Disorders and Schizophrenia. Archives of General Psychiatry, 35, 837-844.

Endicott, J. (1984). Measurement of Depression in Patients with Cancer. Cancer, 53, 2243-2248.

Engel, G.L. (1985). A psychological setting of somatic disease: the «giving up-give up complex». Proceedings of the Royal Society of Medicine, 1967, 553-555.

Fava, G.A., Freyberger, H.J., Bech, P., Christodoulou, G., Sensky, T., y Theorell, T. (1995). Diagnostic criteria for use in psychosomatic research. Psychotherapy \& Psychosomatics, 63, 1-8.

Frank, J. (1973). Persuasion and haling. Baltimore, MD: John Hopkins University Press.

Gilbert, P., Pehl, J., y Allan, S. (1994). The phenomenology of shame and guilty: an empirical investigation. British Journal Clinical Psychology, 67, 23-36.

Gruenberg, E.M. (1967). The social breakdown syndrome: some origins. American Journal of Psychiatry, 123, 1481-1489.

Holtom, N., y Barraclough. (2000). Is the Hospital Anxiety and Depression Scale (HADS) useful in assessing depression in palliative care? Palliative Medicine, 14, 219-220.

Hotopf, M., Chidgey, J., Addington-Hall, J., y Lan Ly, K. (2002). Depression in advanced diseased: a systematic review. Part 1. Prevalence and case finding. Palliative Medicine, 16, 81-97.

Kathol, R., Mutgi, A., Williams, J., Clamon, G., y Noyes, R. (1990). Diagnosis of Major Depression in Cancer patients according to Four Sets Criteria. American Journal of Psychiatry, 147, 1021-1024.

Kelly, B., y Robertson, M. (2002). Terminally ill cancer patients' wish to hasten death. Palliative Medicine, 16, 339-345.

Kissane, D.W., y Kelly, B.J. (2000). Demoralization, depression and desire for death: problems with the
Dutch guidelines for euthanasia of the mentally ill. Australian and New Zealand Journal of Psychiatry, 34, 325-333.

Kissane, D.W., Clarke, D.M., y Street, A.F. (2001). Demoralization syndrome -a relevant psychiatric diagnosis for palliative care. Journal of Palliative Care, 17, 1221.

Kissane, D.W. (1998). Models of psychological response to suffering. Progressing Palliative Care, 6, 197-204.

Klein, D.F., Gittelman, R., Quitkin, F., y Rifkin, A. (1980). Diagnosis and drug treatment of Psychiatric disorders: adults and children. Baltimore, MD: Williams and Wilkins.

Le Fevre, P., Devereux, J., Smith, S., et al. (1999). Screening for psychiatric illness in palliative care inpatient setting: a comparison between the Hospital Anxiety and Depression Scale and the General Health Questionnaire-12. Palliative Medicine, 13, 399-407.

Lloyd-Williams, M., y Payne, S. (2002) Nurse specialist assessment and management of palliative care patients who are depressed- a study of perceptions and attitudes. Journal of Palliative Care, 18, 270-274.

Lloyd-Williams, M., y Spiller, J. (2003). Which depression screening tools should be used in palliative care? Palliative Medicine, 17, 40-43.

Lloyd-Williams, M., Friedman, T., y Rudd, N. (2001). An analysis of the Validity of the Hospital Anxiety and Depression Scale as a screening tool in patients with advanced metastatic cancer. Journal of Pain and Symptom Management, 22, 990-996.

Lloyd-Williams, M., Friedman, T., y Rudd, N. (2000). Criterion validation of the Edinburgh postnatal depression scale as a screening tool for depression in patients with advanced metastatic cancer. Journal of Pain and Symptom Management, 20, 259-65.

Noyes R. Jr., y Kathol, R.G. (1986). Depression and cancer. Psychiatric Developments, 2, 77-100.

Oneschuk, D., y Fainsinger, R. (2001). Medical and ethical dilemmas when an advanced cancer patient discontinues dialysis. Journal of Palliative Care, 18, 123-126.

Page, R.M., y Cole, G.E. (1992). Demoralization and living alone: outcomes from an urban community study. Psychological Reports, 70, 275-280.

Passik, S.D., Dugan, W., McDonald, M.V., Rosenfeld, B., Theobald, D., y Edgerton, S. (1998). Oncologists' Recognition of Depression in Their Patients With Cancer. Journal of Clinical Oncology, 16, 15941600.

Rickelman, B.L. (2002). Demoralization as a precursor to serious depression. Journal of the American Psychiatric Nurses Association, 8, 9-17.

Schmale, A.H. (1972). Giving up as a final common pathway to changes in health. Advances in Psychosomatic Medicine, 8, 20-40. 
Silverstone, P. (1994). Poor efficacy of the hospital anxiety and depression scale in the diagnosis of major depressive disorder in both medial and psychiatric patients. Journal Psychosomatic Research, 38, 441-450.

Slavney, P.R. (1999). Diagnosing Demoralization in consultation. Psychiatry. Psychosomatics, 40, 325-329.

Spitzer, R.L., Endicott, J., y Robins, E. (1978). Research Diagnosis Criteria: rationale and reliability. Archives of General Psychiatry, 35, 773-782.

Spitzer, R.L., Gibbon, M., y Williams, J.B.W. (1996). En W.Breitbart, B. Rosenfeld, H. Pessin, M. Kaim, J.
Funesti-Esch, M. Galietta, C.J. Nelson, y R. Brescia (2000), Depression, hopelessness, and desire for hastened death in terminally ill patients with cancer. JAMA: Journal of the American Medical Association, 284, 2907-2911.

Zatura, A., Guarnaccia, C.A., y Reich, J.W. (1988). Factor structure of mental health measures for older adults. Journal of Consulting and Clinical Psychology, 36, 514-519.

Zigmond, A.S., y Snaith, R.P. (1993). The Hospital Anxiety and Depression Scale. Acta Psychiatrica Scandinavica, 67, 361-370. 